\title{
Quantum Feedback Control for the Deterministic Generation of Schrödinger-Cat States
}

\author{
Masahiro Yanagisawa
}

\begin{abstract}
Continuous measurement and feedback hold tremendous potential for producing non-Gaussian quantum states that are useful for quantum communication and computation. We present a new feedback scheme to deterministically produce quantum non-Gaussian states from linear optical elements and feedback. In our scheme, measurement outcomes are used in two different ways. One implements a multiplicative feedback control which produces nonlinear effects in the system. The other stabilizes the system to produce a desired state deterministically. A design example of the multi-loop feedback structure is demonstrated for the deterministic generation of Schrödinger-cat states as the practical use of quantum feedback control.
\end{abstract}

\section{INTRODUCTION}

Schrödinger-cat states are a typical example of nonGaussian quantum states that are characterized by the negative region of the phase space distribution functions, i.e., Wigner functions. This property represents a significant difference between quantum and classical states because classical distribution functions can never be negative. As in the classical case, quantum linear systems preserve Gaussian properties, so producing non-Gaussian states naturally requires nonlinear quantum effects. Many different physical models have been examined to produce the non-Gaussian states, for example, a trapped ion [1], conditional measurements on a beam splitter [2], one-photon-subtracted squeezed states with $\mathrm{KNbO}_{3}$ [3], [4] and $\mathrm{KTiOPO}_{4}$ [5], ancillaassisted photon subtraction [6]. In particular, the last three methods produces the non-Gaussian states by subtracting photons from squeezed Gaussian states. In the ideal case, a squeezed state is a superposiiton of even photon-number states. The resulting state becomes a superposition of odd photon-number states which is close to Schrödinger-cat states with small coherent amplitudes, and thereby referred to as Schrödinger kittens.

Non-Gaussian states are essential for quantum information technologies and it is important to produce them on demand, but Schrödinger kittens are not sufficient for practical use. In general, the nonlienar effect of optical devices is too weak to produce the non-Gaussian properties. Optical devices tha that we can use to perform reliable operations in experiments are often limited to linear ones. To produce well-controlled, high-quality non-Gaussian states, we need to incorporate advanced engineering in quantum systems.

We propose a deterministic scheme of perfect Schrödingercat state generation using linear optical devices and feedback.

M. Yanagisawa is with Department of Engineering, The Australian National University, Canberra, ACT 0200, Australia y . meanu . edu . au
To produce nonlinear effect from linear devices, we introduce a multiplicative feedback control instead of a standard additive control. In our scheme, feedback is applied to a parameter which is responsible for the interaction between the system and external field. This enables the measurement of nonlinear functions of observables. In particular, it will be shown that from quantum nondemolition (QND) measurement of the position observable $x$ and multiplicative feedback, we can construct QND measurement of $x^{2}$. Under this measurement, the system asymptotically converges to one of the eigenstates of $x^{2}$, which is a Schrödinger-cat state.

The multiplicative feedback control is not sufficient for the deterministic generation of Schrödinger-cat states. So we introduce a multi-loop feedback structure in which measurement outcomes are also used for an additive control. First we feed the measurement outcomes back to the multiplicative process which produces a projection effect that drives the system to one of the Schrödinger-cat states. Then, the measurement outcomes are used again to obtain the estimates of system observables which are fed back to a system Hamiltonian to produce a desired Schrödinger-cat state with high probability, see Fig. 1.

It will be shown that the control design of the deterministic Schrödinger-cat state generation has the same features as the deterministic entanglement generation [7]. Feedback is a control method by which the system is stabilized against unidentified disturbances. It has a significant advantage in improving robustness to uncertainties. In our treatment, stochastic noise in the system under QND measurement is treated as uncertainty and feedback is designed to stabilize the system against it. This property of feedback leads to relaxations to simplify the design of stabilizing feedback controllers for the stochstic nonlinear system. It is supported from the fact that stochastic noise in the system under QND measurement asymptotically disappears and its conditional evolution becomes deterministic.

We start with the description of the system and multiplicative feedback in Sec. II. A rigorous treatment of quantum multiplicative feedback is given by a general formulation of the input-output relation of the system. We will obtain recursive equations to determine a Hamiltonian of the system from the input-output relation. In Sec. III, the stochastic master equation is given to describe the conditional evolution of the system under measurement. This is used to design stabilizing feedback for the deterministic generation of Schrödinger-cat states in Sec. IV. We will consider two different types of feedback. One is a replacement control which controls the system by replacing the state on the phase space depending 


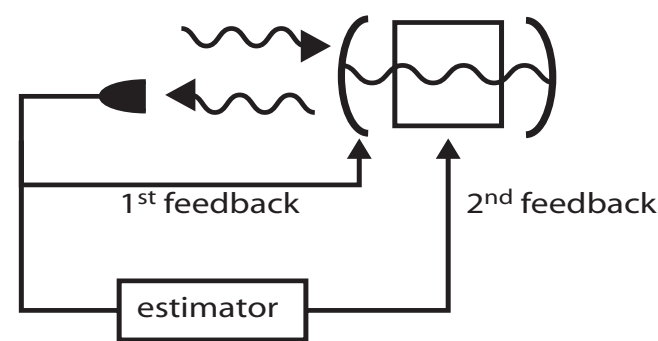

Fig. 1. The schematic representation of the multi-loop feedback. The input and output are split by optical isolators. The output is detected by homodyne measurement and the measurement outcomes are fed back to the interaction between the cavity and the field (the first feedback). The same measurement outcomes are also sent to an estimator which produces the estimate of the cavity state, and then, the estimate is used to modify the system Hamiltonian of the cavity (the second feedback).

on the estimate of the system. The other is based on a universal squeezer by which the state is squeezed depending on the estimate. For both cases, we give numerical examples which show the multi-loop feedback structure successfully produce a desired Schrödinger-cat state with probability one.

\section{THE FIRST FEEDBACK}

A cavity is a quantum optical system that is described by two Hermitian operators $x, y$ satisfying

$$
[x, y]=2 \mathrm{i} \text {. }
$$

These two operators are called $x, y$-quadratures and correspond to the position and momentum of the cavity mode, respectively. The cavity interacts with the external optical field described by a field operator $a$. This field can be thought of as noise to the (cavity) system. In fact, the field operator $a$ or $d A \equiv a d t$ satisfies

$$
d A d A^{\dagger}=d t, \quad d A^{\dagger} d A=0,
$$

which can be thought of as the quantum analog of Ito's lemma.

Let $L$ be a system operator. As stated above, the system is described by $x, y$ so that $L$ is an operator-valued function of $x, y$, i.e., $L=L(x, y)$. Assume the interaction Hamiltonian between the system and the field as

$$
H_{I}=\mathrm{i}\left(L^{\dagger} k a-a^{\dagger} k L\right)
$$

where $k$ is a coupling coefficient. Here we assume that $k$ is an operator. The output of the system is defined by the infinitesimal evolution of the field. From the Hamiltonian (3), it is given as

$$
d A_{\text {out }}=d A+L^{\dagger}[k, d A] d A-d A^{\dagger}[k, d A] L+d t k L .
$$

We make a measurement on the real part of the output (homodyne measurement) so that the measurement outcomes are expressed as

$$
d z=d A_{\text {out }}+d A_{\text {out }}^{\dagger}
$$

We shall introduce two different types of feedback controls. One is the multiplicative feedback and the other is a standard estimate feedback. In this section, we consider the multiplicative one. In this feedback process, the coupling coefficient $k$ is modified depending on the measurement outcomes $z$. This can be experimentally implemented by using a fast rotating half wave plate or placing a lockingadjustable cavity between the system and the field. Assume that the feedback is of the form

$$
k=k_{0}+\epsilon \frac{d z}{d t},
$$

where $1 \ll k_{0}$ is a constant and $\epsilon \ll 1$. This indicates that the coupling coefficient is weakly modified by the measurement outcomes. According to this feedback, the output itself will be modified as well. Suppose that the output is of the form

$$
d A_{\text {out }}=d A+k_{0} \sum_{l=0} \epsilon^{l} X_{l} d t,
$$

where $X_{l}$ are operators to be determined from the fact that the output (7) is consistent with (4). Thus, we have

$$
\sum_{l=0} \epsilon^{l} X_{l}=\left[I+\sum_{l=0} \epsilon^{l+1}\left(X_{l}+X_{l}^{\dagger}\right)\right] L
$$

where we have ignored small terms. Comparing both sides in terms of $\epsilon$, we have

$$
\begin{aligned}
& X_{0}=L \\
& X_{1}=\left(L+L^{\dagger}\right) L \\
& X_{2}=\left\{\left(L+L^{\dagger}\right) L+L^{\dagger}\left(L+L^{\dagger}\right)\right\} L,
\end{aligned}
$$

and other terms can be determined recursively. The resulting coupling coefficient $k$ is given by

$$
k=k_{0}+k_{0} \sum_{l=0} \epsilon^{l+1}\left(X_{l}+X_{l}^{\dagger}\right)
$$

Thus, the interaction Hamiltonian after the feedback is given by

$$
\begin{aligned}
H_{I}= & \mathrm{i} k_{0}\left(L^{\dagger} a-a^{\dagger} L\right) \\
& +\mathrm{i} k_{0} \epsilon\left[L^{\dagger}\left(X_{0}+X_{0}^{\dagger}\right) a-a^{\dagger}\left(X_{0}+X_{0}^{\dagger}\right) L\right] \\
& +\mathrm{i} k_{0} \epsilon^{2}\left[L^{\dagger}\left(X_{1}+X_{1}^{\dagger}\right) a-a^{\dagger}\left(X_{1}+X_{1}^{\dagger}\right) L\right]+\cdots .
\end{aligned}
$$

\section{STOCHASTIC MASTER EQUATION}

Before considering the second feedback, we derive the stochastic master equation which describes the conditional evolution of the system under the measurement (5). For simplicity, we consider up to the first order of the parameter $\epsilon$. Furthermore, for the purpose of applications considered later, we assume that the system operator $L$ is the $x$ quadrature of the cavity mode, i.e., $L=x$. Now the interaction Hamiltonian is expressed as

$$
\begin{aligned}
H_{I} & =\mathrm{i}\left[x\left(k_{0}+2 k_{0} \epsilon x\right) a-a^{\dagger}\left(k_{0}+2 k_{0} \epsilon x\right) x\right] \\
& =\mathrm{i}\left[X a-a^{\dagger} X\right],
\end{aligned}
$$


where

$$
X=k_{0}(1+2 \epsilon x) x .
$$

For an arbitrary system operator $R=R(x, y)$, the quantum stochastic differential equation is given by

$$
\begin{aligned}
d R & =(-\mathrm{i}[H, R]+\mathcal{L}(X) R) d t+[X, R]\left(d A+d A^{\dagger}\right), \\
d A_{\text {out }} & =X d t+d A,
\end{aligned}
$$

where $\mathcal{L}(X) R=X R X-\left(X^{2} R+R X^{2}\right) / 2$ and $H$ is an additional Hamiltonian describing the second feedback.

Let us introduce the change of coordinates

$$
\begin{gathered}
x+\frac{1}{4 \epsilon} \rightarrow x, \\
X+\frac{k_{0}}{8 \epsilon} \rightarrow X .
\end{gathered}
$$

Note that the stochastic differential equation is invariant under this replacement, i.e., the stochastic differential equation after the change of coordinates is the same as (17). (The output (17b) should be modified by the change of coordinates. However, it can be easily seen that the deterministic replacement of the output equation does not influence the conditional expectation that is what we want for the second feedback.)

Denote by $\pi(R)$ the conditional expectation of the operator $R$ when we obtain the measurement outcomes $z$ given by (5). The stochastic master equation for $\pi(R)$ is calculated as

$$
\begin{aligned}
d \pi(R)= & \pi(-\mathrm{i}[H, R]+\mathcal{L}(X) R) d t \\
& +[\pi(X R+R X)-\pi(2 X) \pi(R)] d w,
\end{aligned}
$$

where $d w=d z-\pi(X) d t$ is the innovation process.

\section{THE SECOND FEEDBACK}

In this section, we consider the second feedback loop to stabilize the system. Two different types of feedback will be introduced. One is replacement control which produces asymmetric control inputs and the other is based on a universal squeezer which is a symmetric control.

\section{A. Relaxations}

The second feedback of our multi-loop scheme is aimed at for the deterministic generation of Schrödingercat states. The multiplicative feedback control can produce Schrödinger-cat states, but the outcome is still probabilistic and we do now know which Schrödinger-cat state is obtained. To make the system deterministic, we design the system Hamiltonian depending on the estimate of the system, i.e., $H=H(\pi(R))$, which stabilizes the system to converge to a single state asymptotically. The design of such controllers is limited to a fundamental technique from Lyapunov theory, but the search of Lyapunov functions is not easy for nonlinear stochastic systems. Here we introduce relaxation based on the robustness of feedback and simplify the design of controllers, as considered for the deterministic photon number state and entanglement generation [7]. The stochastic master equation (20) describes the QND measurement of $x^{2}$. The advantage of using QND measurement for control is that the measured observable becomes deterministic asymptotically. In fact, since the strength of the stochastic term in (20) is determined by the variance which goes to zero under QND measurement, the stochastic noise is attenuated over time. Thus, regarding the stochastic term of (20) as small uncertainties in the system and utilizing the robustness of feedback control, one can expect that the system under QND measurement is controlled by designing controllersfor the deterministic terms in (20).

\section{B. Replacement Control}

Let us consider a case where the control Hamiltonian is of the form

$$
H=-\frac{u}{2} y
$$

where $u$ is an input function determined by the conditional expectation. This Hamiltonian represents the replacement of a quantum state on the phase space.

As stated above, we analyze the stability of the system only for the deterministic part of (20). It is given by

$$
\begin{aligned}
\frac{d \pi(x)}{d t} & =u, \\
\frac{d \pi(X)}{d t} & =4 \epsilon k_{0} u \pi(x) .
\end{aligned}
$$

To produce Schrödinger-cat states, we stabilize $X$ at $\bar{X}=$ $2 \epsilon k_{0} \bar{x}^{2}$, where $\bar{x}$ is a given constatnt. Then, we expect that the resulting state is

$$
\alpha|+\bar{x}\rangle+\beta|-\bar{x}\rangle \cdot \quad\left(|\alpha|^{2}+|\beta|^{2}=1\right)
$$

These states are eigenstates of the observable $x^{2}$ with eigen value $\bar{x}^{2}$.

Let us consider an input of the form

$$
u=c(\pi(X)-\bar{X}),
$$

where $c$ is a constant, and a Lyapunov function

$$
\dot{V}=[\pi(X)-\bar{X}]^{2} \text {. }
$$

Assume that we start control from the vacuum state in the $x$ coordinate. Since $\pi(X)<\bar{X}$ at the initial time,

$$
\left\{\begin{array}{l}
c>0 \Rightarrow u<0 \Rightarrow \pi(x)<0 \\
c<0 \Rightarrow u>0 \Rightarrow \pi(x)>0
\end{array}\right.
$$

In either case, we have $\dot{V}=(\pi(X)-\bar{X}) 4 \epsilon k_{0} u \pi(x)<0$, so the system is stabilized at $\bar{X}$.

This analysis is very similar to the deterministic photon number state generation [7] and the input (25) can successfully produce Schrödinger-cat states. Unlike the photon number case, however, the form (21) breaks the symmetry of the system. While the symmetry of the final state is guaranteed by QND measurement in the case of the deterministic photon number generation, the resulting state can be asymmetric in the case of Schrödinger-cat states. Although we can obtain 


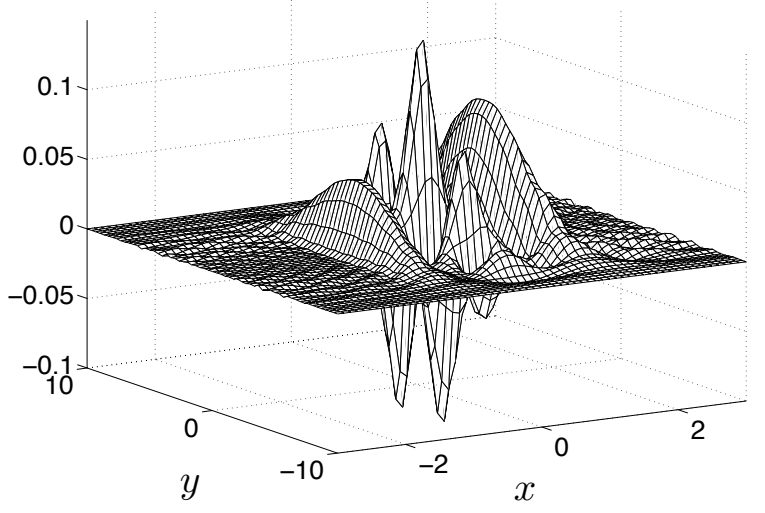

Fig. 2. The $\mathrm{W}$ function of a Schrödinger-cat state produced by the input (25). As the control input is asymmetric, the resulting $\mathrm{W}$ function is also asymmetric with respect to $x$. Around the center $(x=0)$, the $\mathrm{W}$ function has the negative regions.

a Schrödinger-cat state (24) with the control input (25), we still do not know which Schrödinger-cat state (or what coefficient) is obtained.

\section{Universal Squeezer}

To obtain a desired symmetric (i.e., $\alpha=\beta$ ) Schrödingercat state deterministically, we use a universal squeezer [8] [9] described by a system Hamiltonian

$$
H=-\frac{u}{8}(x y+y x) .
$$

This Hamiltonian squeezes the quantum state to the $x$ or $y$ direction in the phase space.

In this case, the deterministic part of (20) is simply given by

$$
\frac{d \pi\left(x^{2}\right)}{d t}=\pi\left(x^{2}\right) u
$$

Consider a Lyapunov function

$$
V=\frac{1}{2}\left[\pi\left(x^{2}\right)-\bar{x}^{2}\right]^{2},
$$

then,

$$
\dot{V}=\left[\pi\left(x^{2}\right)-\bar{x}^{2}\right] \pi\left(x^{2}\right) u .
$$

Since $\pi\left(x^{2}\right)>0$, a control input

$$
u=-K\left[\pi\left(x^{2}\right)-\bar{x}^{2}\right] \pi\left(x^{2}\right) \quad(K>0)
$$

results in $\dot{V}<0$ except for the target point $\pi\left(x^{2}\right)=\bar{x}^{2}$ and therefore stabilizes the system at $\bar{x}^{2}$. In this case, we obtain a symmetric Schrödinger-cat state

$$
\frac{1}{\sqrt{2}}(|+\bar{x}\rangle+|-\bar{x}\rangle)
$$

Figure 4 shows fidelity to a cat state with $\bar{x}=2$ for the system initially prepared in a vacuum state and the corresponding input $u$. At the beginning, we need a relatively

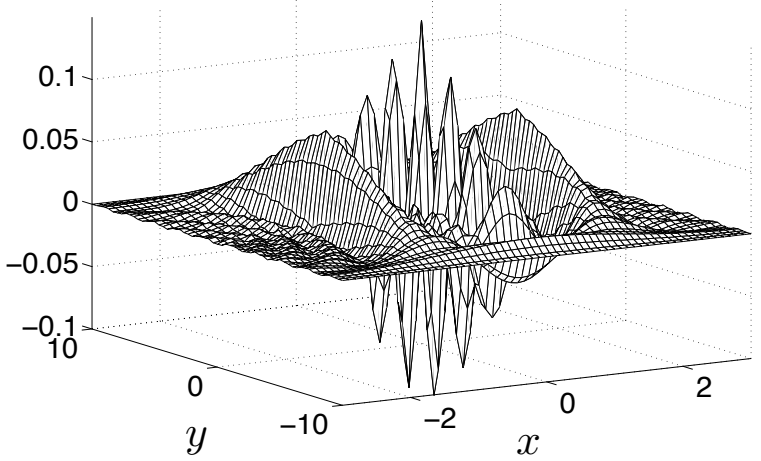

Fig. 3. The $\mathrm{W}$ function of a Schrödinger-cat state produced by the universal squeezer and the control input (31). In this case, the resulting $\mathrm{W}$ function is symmetric with respect to $x$. This is actually equivalent to the Shcrödingercat state $(|+2\rangle+|-2\rangle) / \sqrt{2}$ as the fidelity shows in Fig. 4.
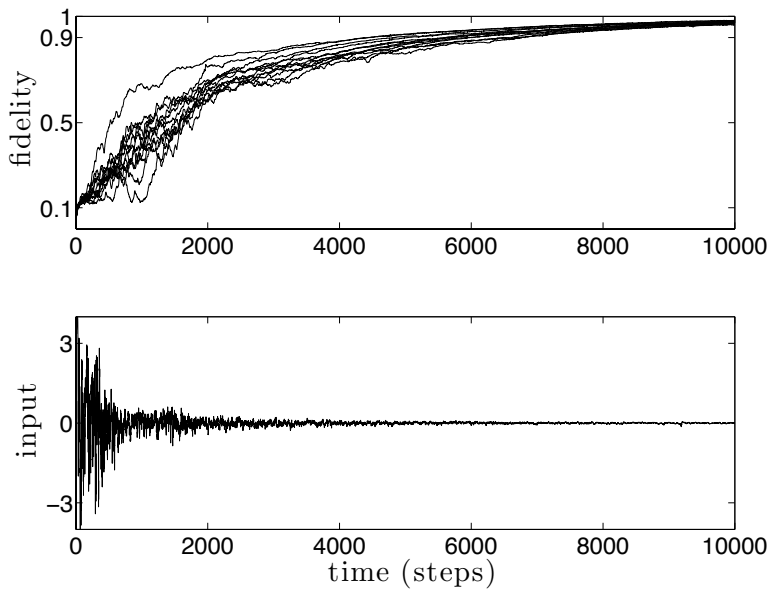

Fig. 4. (A) Fidelity to a symmetric Schrödinger-cat state $(|+2\rangle+$ $|-2\rangle) / \sqrt{2}$ (10 trajectories) subject to the control $(27,31)$. (B) The corresponding control input $u$.

strong control input to drive the system to a target cat state. However, once the system gets close to the target state (fidelity $\sim 0.5$ ), the input is no loger required and the projection effect of the measurement drives the system to the target state.

This feedback control can be implemented more efficiently if we introduce squeezing to the initial state because in the case of Fig.4, the input (31) is designed to amplify the noise of $x$-quadrature at the early stage of control. A squeezing operator is given by

$$
S(r)=e^{-\frac{\mathrm{i} r}{4}(x y+y x)},
$$

where $r$ is a squeezing parameter. Since

$$
\left\langle 0\left|S(r)^{\dagger} x^{2} S(r)\right| 0\right\rangle=e^{2 r}
$$

we have to set the initial squeezed state as $e^{r}=\bar{x}$, i.e., an 
appropriate initial squeezing parameter is given by

$$
r=\log \bar{x} .
$$

Then, Schrödinger-cat states can be obtained with weaker control inputs effectively.

\section{CONCLUSION}

We have developed a new feedback scheme for the construction of nonlinear measurements from linear optical devices. This scheme consists of two different types of feedback. One is multiplicative feedback and the other is estimate feedback. The necessary nonlinearity to produce non-Gaussian states is provided by the multiplicative action in the first feedback loop. The second feedback is used to stabilize the system for the deterministic non-Gaussian state generation. For the design of the second loop, we introduced the replacement control and the universal squeezer. In both cases, the resulting phase space distribution has the negative region. This is a distinguishing feature of quantum systems and cannot be observed in the classical case. It is worth noting that while the replacement control breaks the symmetry of the system, the universal squeezer results in symmetric Schrödinger-cat states. Thus, the universal squeezer is more useful for the deterministic geneation of Schrödinger-cat states.

\section{REFERENCES}

[1] C. Monroe, D. M. Meekhof, B. E. King and D. J. Wineland, A "Schrödinger Cat" Superposition State of an Atom, Science 272, 11311136 (1996).

[2] M. Dakna, T. Anhut, T. Opatrný, L. Knöll and D.-G. Welsch, Generating Schrödinger-cat-like states by means of conditional measurements on a beam splitter, Phys. Rev. A 55, 3184-3194 (1997).

[3] A. Ourjoumtsev, R. Tualle-Brouri, J. Laurat and P. Grangier, Generating Optical Schrödinger Kittens for Quantum Information Processing, Science 312, 83-86 (2006).

[4] J. S. Neergaard-Nielsen, B. Melholt Nielsen, C. Hettich, K. Mølmer and E. S. Polzik, Phys. Rev. Lett. 97083604 (2006).

[5] K. Wakui, H. Takahashi, A, Furusawa and M. Sasaki, Photon subtracted squeezed states generated with periodically poled $\mathrm{KTIOPO}_{4}$, Opt. Exp. 15 3568-3574 (2007).

[6] H. Takahashi, K. Wakui, S. Suzuki, M. Takeoka, K. Hayasaka, A. Furusawa and M. Sasaki, Phys. Rev. Lett. 101233605 (2008).

[7] M. Yanagisawa, Quantum Feedback Control for Deterministic Entangled Photon Generation, Phys. Rev. Lett. 97190201 (2006).

[8] J. Yoshikawa, T. Hayashi, T. Akiyama, N. Takei, A. Huck, U. L. Andersen and A. Furusawa, Demonstration of deterministic and high fidelity squeezing of quantum information, Phys. Rev. A 76 060301(R) (2007).

[9] R. Filip, P. Marek and U. L. Andersen, Measurement-induced continuous-variable quantum interactions, Phys. Rev. A 71042308 (2005). 